\title{
Interleukin-27 Ameliorates Renal Ischemia-Reperfusion Injury through Signal Transducers and Activators of Transcription 3 Signaling Pathway
}

\author{
Peihui Zhou ${ }^{a}$ Bo Deng ${ }^{a} \quad$ Ming Wu $^{\text {b }}$ Feng Ding ${ }^{a} \quad$ Li Wang $^{\text {a }}$ \\ aDepartment of Nephrology, Shanghai Ninth People's Hospital, Shanghai Jiao Tong \\ University School of Medicine, Shanghai, PR China; ${ }^{b}$ Department of Nephrology, Shuguang \\ Hospital Affiliated to Shanghai University of Traditional Chinese Medicine, TCM Institute of \\ Kidney Disease of Shanghai University of Traditional Chinese Medicine, Key Laboratory of \\ Liver and Kidney Diseases, Ministry of Education, Shanghai Key Laboratory of Traditional \\ Chinese Clinical Medicine, Shanghai, PR China
}

\section{Keywords}

Interleukin-27 · Renal ischemia-reperfusion injury · Acute kidney injury · Signal transducer and activator of transcription $3 \cdot$ Apoptosis

\begin{abstract}
Background: Acute kidney injury (AKI) is a clinical syndrome characterized by significant morbidity and a high death rate. Interleukin (IL)-27 is a newly described member of the IL-6/IL-12 heterodimeric cytokine family and displays anti-inflammatory and antiapoptotic properties. Objectives: To determine the effect and mechanism of IL-27 in AKI. Method: We used a mouse model of renal ischemia/reperfusion (I/R) injury to investigate whether IL-27 has a therapeutic potential for the treatment of AKI. For the IL-27 administration group, IL-27 protein was injected $1 \mathrm{~h}$ before ischemia. Human proximal tubular epithelial cells were exposed to ischemia for $2 \mathrm{~h}$ and followed by $2 \mathrm{~h}$ of reperfusion (I2h+R2h treatment) used as an in vitro model to investigate the effect of IL-27. Results: Two IL-27 subunits, Epstein-Barr virus gene 3 and p28, were upregulated in kidneys $24 \mathrm{~h}$ after I/R. Renal expression of IL-27 receptor subunits (gp130 and WSX-1) was also increased. Treatment with IL-27 reduced structural/functional damages, ameliorated renal inflammation, inhibited the cleaved caspase-3 expression, upregulated antiapoptotic protein $\mathrm{Bcl}-2$ and downregulated proapoptotic protein Bax in the kidneys of mice subjected to I/R. Meanwhile, the level of IL-27 receptor on renal tubular epithelial cells was
\end{abstract}

Peihui Zhou and Bo Deng contributed equally to the research. 


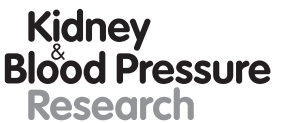

Research \begin{tabular}{l|l}
\hline Kidney Blood Press Res 2019;44:1453-1464 \\
\hline DOI: 10.1159/000503923 & $\begin{array}{l}\text { @ 2019 The Author(s). Published by S. Karger AG, Basel } \\
\text { www.karger.com/kbr }\end{array}$ \\
\hline
\end{tabular}

Zhou et al.: IL-27 in AKI

increased after $12 \mathrm{~h}+\mathrm{R} 2 \mathrm{~h}$ treatment, and IL-27 administration suppressed $12 \mathrm{~h}+\mathrm{R} 2 \mathrm{~h}$-induced epithelial cell apoptosis. Furthermore, IL-27 treatment led to activation of signal transducer and activator of transcription 3 (STAT3) both in vivo and in vitro, and IL-27-mediated protection against I $2 \mathrm{~h}+\mathrm{R} 2 \mathrm{~h}$ injury was abolished by STAT3 inhibition. Conclusions: IL-27 protects against renal I/R injury by activating STAT3, suggesting that IL-27 may represent a novel strategy for the treatment of AKI.

(c) 2019 The Author(s)

Published by S. Karger AG, Basel

\section{Introduction}

Acute kidney injury (AKI) is a clinical syndrome characterized by significant morbidity and a high death rate, which occurs in $2-7 \%$ of the hospitalized population [1]. The incidence of hospital-acquired AKI is increasing and is imposing an economic burden on health care expenditures. Despite the recent advances in basic research, clinically therapeutic approaches for AKI are still not available, and the management is limited to supportive care, such as renal replacement therapy [2]. Therefore, novel therapies are urgently needed for preventing or minimizing AKI. The primary clinical causes of AKI include reduced renal perfusion, nephrotoxic drug effect and sepsis, among which ischemic reperfusion (I/R) injury is the most frequent reason [3]. Its pathogenesis includes inflammatory responses, apoptosis and necrosis in renal tubular cells. Several genes, such as the proapoptotic gene Bax and the antiapoptotic gene Bcl-2 also play essential roles.

Interleukin-27 (IL-27) is a newly described molecule in the IL-6/IL-12 family consisting of two subunits, Epstein-Barr virus gene 3 (EBI3) and p28 [4]. Antigen-presenting cells and endothelial cells are major sources of IL-27 production in response to host- or pathogenderived inflammatory cues [5]. IL-27 transmits signals through a high-affinity receptor complex consisting of the IL-27 receptor (WSX-1) and gp130 [6]. WSX-1 is the unique receptor for IL-27, whereas gp 130 also senses signals from other cytokines including IL- 6 and is widely expressed on immune and nonimmune cells. IL-27 shows suppressive effects on T-cell functions or differentiation and has recently been described as an immunosuppressive cytokine [7]. In addition, recent studies showed that IL-27 displays an antiapoptotic effect by predominantly activating signal transducer and transcription factor 3 (STAT3) [8]. It is noteworthy that treatment with exogenous IL-27 induced inhibitory immune responses and tissue protection in disorder models of myocardial I/R injury [9], myocarditis [10], osteoarthritis [11], lung inflammation [12] and rheumatoid arthritis [13]. These studies revealed the therapeutic potential of IL-27 in inflammatory disorders.

However, the role of IL-27 in AKI has not been reported. Here, we used a mouse model of renal I/R injury to investigate whether IL-27 has a therapeutic potential for the treatment of AKI.

\section{Materials and Methods}

\section{Animals}

All animal experiments were carried out in accordance with Institutional Animal Care and Use Committee guidelines. In this study, wild-type male mice 6-8 weeks old (approx. 21 g) with C57BL/6J background were provided by Shanghai Jiao Tong University 9th Hospital Center laboratory. After anesthesia, an incision was made into the abdominal cavity to expose the kidneys, and renal I/R was imposed by clamping bilateral renal pedicles for $35 \mathrm{~min}$. For sham-operated mice, kidney pedicles were subjected to the same procedure but were not clamped. Mice were randomized into four groups: sham, sham with recombinant IL-27 
Table 1. Sequences of real-time PCR primers

\begin{tabular}{|c|c|}
\hline Gene name & Primer sequence \\
\hline Mouse EBI3 & $\begin{array}{l}\text { Forward, 5'-GTCCTTCATTGCCACKTACAGGCT-3' } \\
\text { Reverse, 5' -GGAGGGTCSGGCTTGATGAT-3' }\end{array}$ \\
\hline Mouse p28 & $\begin{array}{l}\text { Forward, 5'-AGGGARTTCACAGTCAGCCTG-3' } \\
\text { Reverse, 5'-GCCTGGAARGTCAGGGAAACAT-3' }\end{array}$ \\
\hline Mouse gp130 & $\begin{array}{l}\text { Forward, 5'-ATACCTTAAACAAGCTCCACCTTC-3' } \\
\text { Reverse, 5' } \text { 5'AGTTTCATTTCCAATGATGGTTCT-3' }^{\prime}\end{array}$ \\
\hline Mouse WSX-1 & $\begin{array}{l}\text { Forward, 5'-GAAACCCAAATGAAGCCAAA-3' } \\
\text { Reverse, 5'-GCCTCCTGACATCTTCGGTA-3' }\end{array}$ \\
\hline Mouse TNF- $\alpha$ & $\begin{array}{l}\text { Forward, 5'-CAGGCGGTGCCTATGTCTC-3' } \\
\text { Reverse, 5'-CGATCACCCCGAAGTTCAGTAG-3' }\end{array}$ \\
\hline Mouse IL-17A & $\begin{array}{l}\text { Forward, 5'-TCAGCGTGTCCAAACACTGAG-3' } \\
\text { Reverse, 5'-CGCCAAGGGAGTTAAAGACTT-3' }\end{array}$ \\
\hline Mouse IL-10 & $\begin{array}{l}\text { Forward, 5'-GCTCTTACTGACTGGCATGAG-3' } \\
\text { Reverse, 5'-CGCAGCTCTAGGAGCATGTG-3' }\end{array}$ \\
\hline Mouse IL-6 & $\begin{array}{l}\text { Forward, 5'-TAGTCCTTCCTACCCСAATTTCC-3' } \\
\text { Reverse, 5'-TTGGTCCTTAGCCACTCCTTC-3' }\end{array}$ \\
\hline Mouse $\beta$-actin & $\begin{array}{l}\text { Forward, 5'-GGCTGTATTCCCCTCCATCG-3' } \\
\text { Reverse, 5'-CCAGTTGGTAACAATGCCATGT-3' }\end{array}$ \\
\hline Human gp130 & $\begin{array}{l}\text { Forward, 5'-CCGTGTGGTTACATCTACCCT-3' } \\
\text { Reverse, 5'-CGTGGTTCTGTTGATGACAGTG-3' }\end{array}$ \\
\hline Human WSX-1 & $\begin{array}{l}\text { Forward, 5'-TCTGTCAGTTCCGGTACAAGG-3' } \\
\text { Reverse, 5'-GGTTCTGCATCTCAACAGGAGT-3' }\end{array}$ \\
\hline Human $\beta$-actin & $\begin{array}{l}\text { Forward, 5'-AAGGAGCCCCACGAGAAAAAT-3' } \\
\text { Reverse, 5'-ACCGAACTTGCATTGATTCCAG-3' }\end{array}$ \\
\hline
\end{tabular}

protein, I/R with vehicle, and I/R with recombinant IL-27 protein. For the IL-27 administration group, the recombinant mouse IL-27 protein $(1 \mu \mathrm{g} /$ mouse, 577402, BioLegend, CA, USA) was injected $1 \mathrm{~h}$ before ischemia. We selected this dose based on previously published studies [14-16]. After 6, 12, 24, 48 and $72 \mathrm{~h}$ of reperfusion, the blood and kidney were collected for further analysis. An autoanalyzer (ADVIA2400, Siemens, Shanghai, China) was used to analyze blood urea nitrogen (BUN) and serum creatinine levels ( $\mathrm{SCr}$ ).

\section{RNA Extraction and Quantitative Real-Time Polymerase Chain Reaction}

To analyze p28, EBI3, gp130, WSX-1, IL-17A, TNF- $\alpha$, IL-10 and IL-6 gene expression, total RNA was extracted from the whole kidneys or cells using TRIzol (15596-026, Thermo Fisher Scientific, MA, USA). Total RNA ( $1 \mu \mathrm{g})$ was reverse transcribed using PrimeScript RT reagent kit (RR037A, TaKaRa Biotechnology, Kyoto, Japan). The resulting 50 ng of single-stranded cDNA of each sample was subjected to quantitative real-time polymerase chain reaction (RT-PCR). Real-time data were normalized against $\beta$-actin, and the relative change in mRNA expression was counted using the $2^{-\Delta \Delta \mathrm{C}_{\mathrm{T}}}$ method. The primer sequences are given in Table 1 .

\section{Immunoblotting}

Protein from kidney tissues or cells was extracted using RIPA buffer (P0013B, Beyotime, Shanghai, China). After assessing the concentration of protein with BCA Protein Quantifi- 


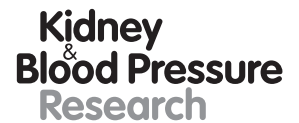

Kidney
Blood Pressure
Research \begin{tabular}{l|l}
\hline Kidney Blood Press Res 2019;44:1453-1464 \\
\hline DOI: 10.1159/000503923 & $\begin{array}{l}\text { @ 2019 The Author(s). Published by S. Karger AG, Basel } \\
\text { www.karger.com/kbr }\end{array}$ \\
\hline
\end{tabular}

Zhou et al.: IL-27 in AKI

cation kits (P0012, Beyotime, Shanghai, China), protein samples were mixed with the sodium dodecylsulfate loading buffer (P0015L, Beyotime, Shanghai, China) and were further separated by sodium dodecylsulfate-polyacrylamide gels, which were transferred to polyvinylidene fluoride membranes for immunoblotting. After blocking, blots were incubated with specific primary antibodies as follows: Bcl-2 (ab182858, Abcam, MA, USA), Bax (ab32503, Abcam, MA, USA), STAT3 (ab68153, Abcam, MA, USA), phosphorylated STAT3 (pSTAT3; ab76315, Abcam, MA, USA), cleaved caspase-3 (9664, Cell Signaling Technology, Danvers, MA, USA) and $\beta$-actin (60008-1-Ig, Proteintech, PA, USA). Signals were detected using the enhanced chemiluminescence (P36599, Millipore, MA, USA) detection system after further incubation with corresponding horseradish peroxidase-conjugated secondary antibodies. ImageJ was used to quantify the images of Western blots.

\section{Histopathology}

The mouse kidney samples were fixed in formaldehyde, which were further immersed in $80 \%$ ethanol and subsequently embedded in paraffin. Paraffin tissue sections were dewaxed, rehydrated and dyed with periodic acid-Schiff (PAS) according to established protocols. Histological changes were viewed by light microscopy. The quantitative injury evaluation was recorded as described previously [17]: scored 0, normal; 1, below 10\%; 2, 10-25\%; 3, $25 \%-75 \%$; and 4 , over $75 \%$ of the area of interest. For each slide, at least $5-10$ fields $(\times 400$ magnification) were viewed.

\section{Cell Culture and Treatment}

Human proximal tubular epithelial cells (HK-2) were purchased from the cell bank of the Chinese Academy of Sciences. Cells were cultured in Dulbecco's modified Eagle medium/ nutrient mixture F12 medium (DMEM/F12, 11330-032, GIBCO, MA, USA) containing 10\% fetal bovine serum (FBS; 0100147, GIBCO, MA, USA) and 1\% penicillin/streptomycin (03-031-1B, Bioind, Kibbutz Beit Haemek, Israel) in an incubator containing $5 \% \mathrm{CO}_{2}$ at $37^{\circ} \mathrm{C}$. The in vitro model of I/R was carried out as follows: HK-2 cells were washed twice by phosphate-buffered saline, and cells were starved in a glucose-free DMEM (11966-025, GIBCO, MA, USA) in an anoxic chamber with $5 \% \mathrm{CO}_{2}$ and $95 \% \mathrm{~N}_{2}$ for $2 \mathrm{~h}$. Then cells were replaced with DMEM medium containing 10\% FBS and with $21 \%$ oxygen for reperfusion for another $2 \mathrm{~h}$. Control cells were cultured in normal DMEM/F12 medium (11330-032, GIBCO, MA, USA) with $21 \%$ oxygen [18]. To determine the potential effect of IL-27 in the in vitro model of I/R, HK-2 cells were starved for $12 \mathrm{~h}$ with complete DMEM/F12 without FBS, and $50 \mathrm{ng} / \mathrm{mL}$ recombinant human IL-27 protein (NGZ2716111, R\&D Systems Inc., MN, USA) or phosphate-buffered saline were added to the cells at $16 \mathrm{~h}$ before $\mathrm{I} 2 \mathrm{~h}+\mathrm{R} 2 \mathrm{~h}$ exposure according to previous studies [19-21]. For treatment with the STAT3 inhibitor, Stattic $(30 \mu \mathrm{mol} / \mathrm{L}, \mathrm{S} 7024$, Selleck, TX, USA) was added $2 \mathrm{~h}$ prior to the treatment with IL-27.

\section{Flow Cytometry}

Apoptotic cells were detected by flow cytometry using annexin $\mathrm{V} /$ propidium iodide apoptosis detection kit (V13242, Thermo Fisher Scientific, MA, USA).

\section{Statistics}

Data are presented as the mean \pm standard error of the mean (SEM). We use GraphPad Prism version 5.0 software (GraphPad Inc.) to analyze and display the data. Data were analyzed by the 2-tailed $t$ test for two groups when the data were normally distributed. For multiple comparisons, one-way analysis of variance was applied. $p<0.05$ was evaluated to represent significance. 


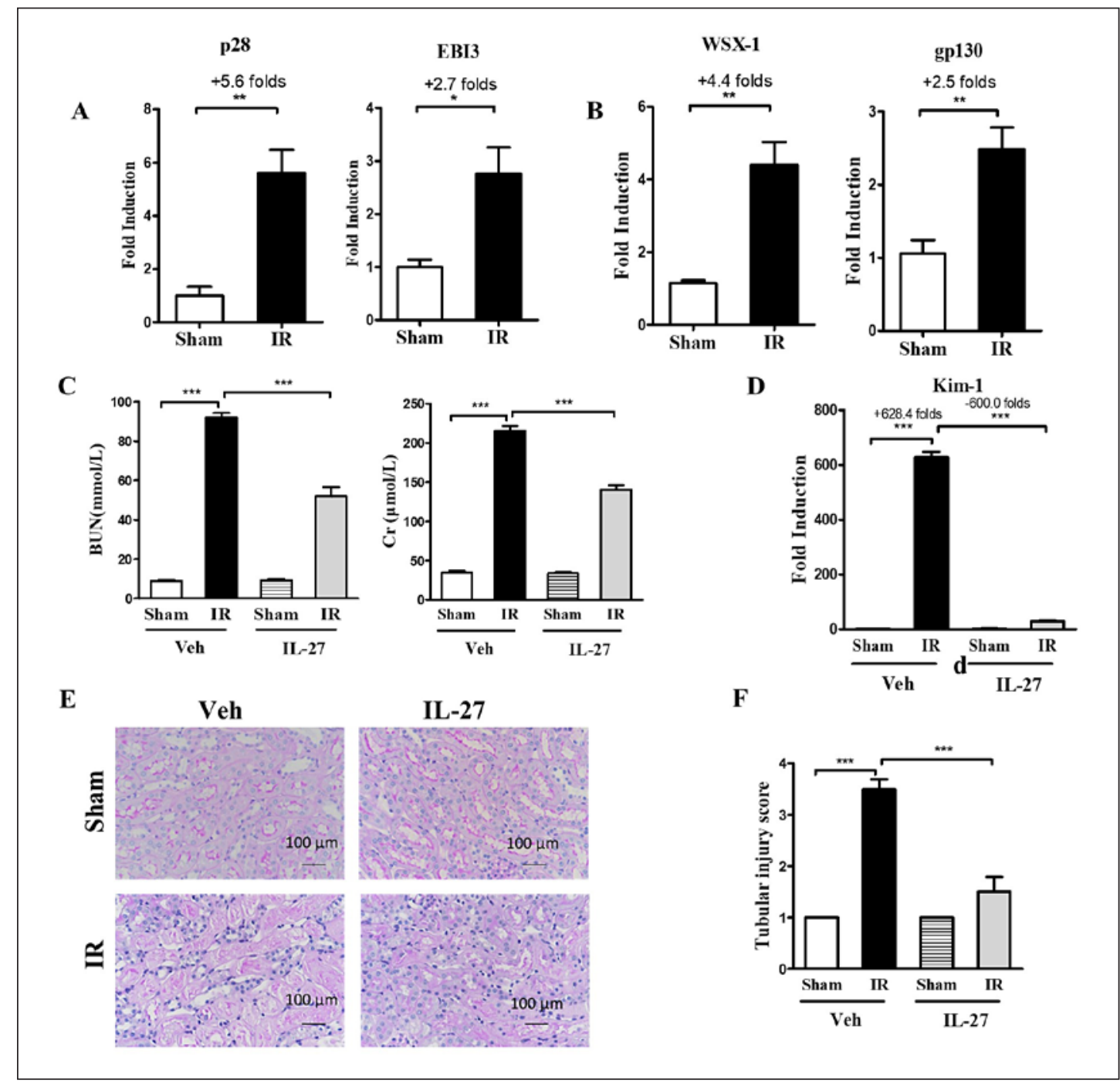

Fig. 1. IL-27 and its receptors were upregulated in kidneys after I/R, and IL-27 treatment prevents renal injury. A The mRNA expression of the two IL-27 subunits, EBI3 and p28, was assessed by real-time polymerase chain reaction (RT-PCR) in kidneys $24 \mathrm{~h}$ after I/R. B The mRNA expression level of the two IL-27 receptors, gp130 and WSX-1, was assessed in kidneys. C An autoanalyzer was used to analyze blood urea nitrogen (BUN) and serum creatinine (Cr) levels. D The mRNA expression of Kim-1 was examined by RT-PCR. E Representative photomicrographs of renal tissues from sham-operated or I/R-treated mice. Original magnification $\times 400$. Scale bar $=100 \mu \mathrm{m}$. $\mathbf{F}$ Quantitative assessment of tubular injury. Data are expressed as means \pm SEM. ${ }^{*} p<0.05,{ }^{* *} p<0.01,{ }^{* * *} p<0.001$. Similar results were obtained in 3 independent experiments with 6-7 mice per group.

\section{Results}

\section{IL-27 and Its Receptors Were Upregulated in Kidneys after 24 h of Reperfusion}

To detect the effects of IL-27 on the renal injury, we used a widely accepted animal model of kidney injury induced by $\mathrm{I} / \mathrm{R}$, which is a common cause of AKI. Figure $1 \mathrm{~A}$ shows that the mRNA expressions of the two IL-27 subunits, EBI3 and p28, were increased in kidneys after $24 \mathrm{~h}$ reperfusion in I/R-induced AKI (approx. 5.6-fold and 2.7-fold, respectively). As shown in online supplementary Figure 1 (for all online suppl. material, see www.karger.com/ 


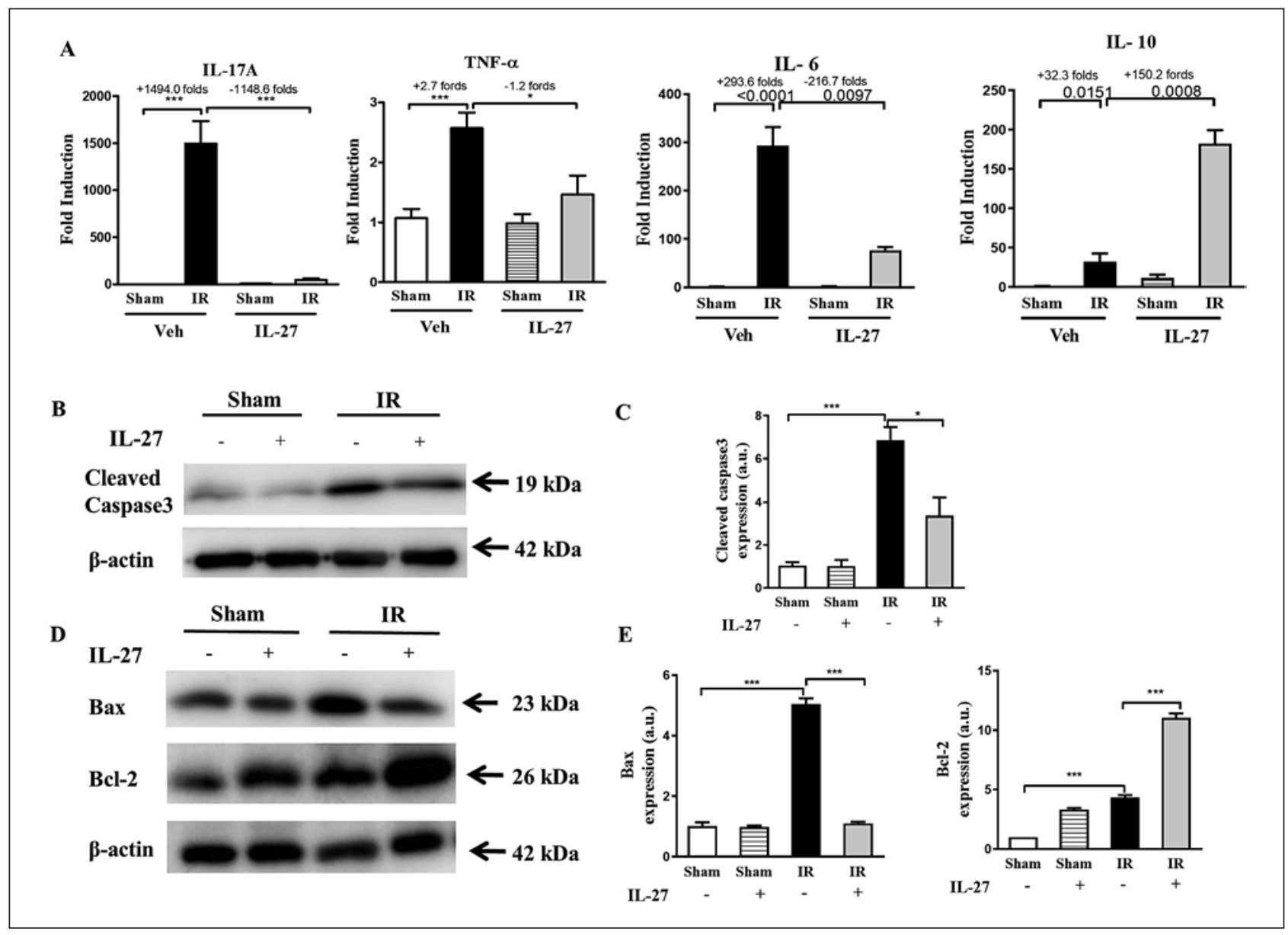

Fig. 2. IL-27 reduces kidney inflammation and apoptosis after I/R. A The relative mRNA levels of the proinflammatory cytokines IL-17A, TNF- $\alpha$, IL-6 and the anti-inflammatory cytokine IL-10. B Representative Western blot images of cleaved caspase-3 in renal tissue. C Densitometric analysis of cleaved caspase-3. Values are expressed as relative expression (arbitrary units). D Representative Western blot images of Bcl-2 and Bax in renal tissue. E Densitometric analysis of Bcl-2 and Bax. Values are expressed as relative expression (arbitrary units). Data are expressed as means \pm SEM. $* p<0.05, * * * p<0.001$. Similar results were obtained in 3 independent experiments with 6-7 mice per group.

doi/10.1159/000503923), EBI3 and p28 mRNA levels were significantly elevated in the kidneys $24 \mathrm{~h}$ after reperfusion, then decreased 48 and $72 \mathrm{~h}$ after reperfusion, which was in parallel with the change of BUN and SCr levels. IL-27 exerts its biological effects through receptor complexes composed of gp130 and WSX-1, the gene expressions of which were also significantly elevated to approximately 4.4- and 2.5-fold, respectively, $24 \mathrm{~h}$ after I/R (Fig. 1B). Together, these data indicate that the expressions of IL-27 and its receptors are increased in the injured kidneys which might play a role in I/R-induced AKI.

\section{IL-27 Treatment Protects against I/R-induced Kidney Injury}

Because the kidney expression of IL-27 receptors was elevated after I/R, we next explored the potential therapeutic effect of IL-27 in AKI. As shown in Figure 1, the renal damages were less pronounced in the I/R group with IL-27 administration than that in the group with vehicle treatment. BUN and SCr levels, two parameters of renal function, were assessed. The IL-27 treatment decreased the levels of BUN and SCr in the I/R group by 46 and 36\%, respectively 


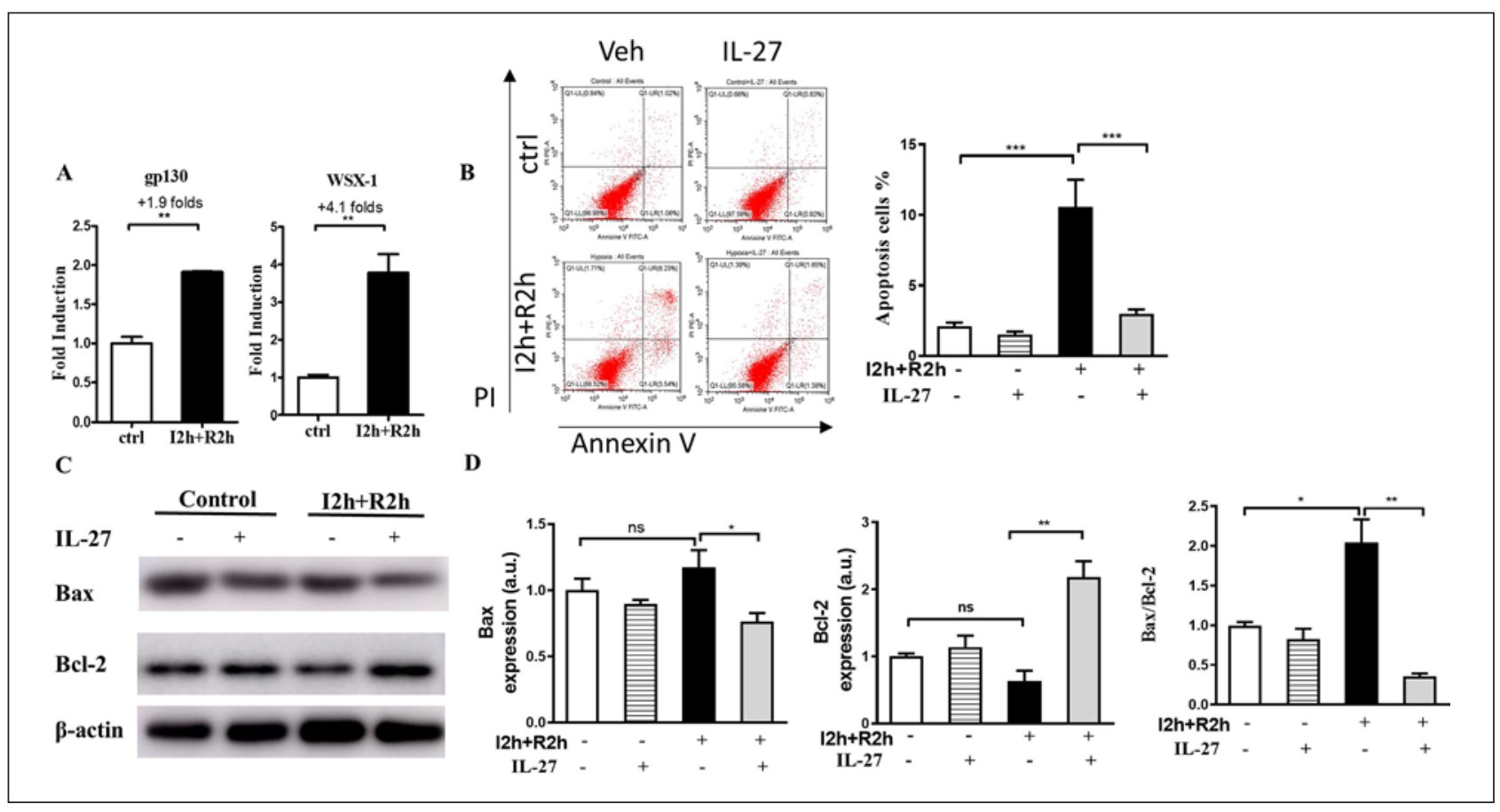

Fig. 3. IL-27 reduced I $2 \mathrm{~h}+\mathrm{R} 2 \mathrm{~h}$-induced apoptosis in renal tubular epithelial cells. The in vitro model of ischemia-reperfusion (I2h+R2h treatment) was carried out as follows: HK-2 cells were washed twice by phosphate-buffered saline and were then kept in a glucose-free DMEM medium in an anoxic chamber with 5\% $\mathrm{CO}_{2}$ and $95 \% \mathrm{~N}_{2}$ for $2 \mathrm{~h}$. Then cells were cultured with $10 \%$ FBS complete medium in $21 \%$ oxygen atmosphere for reperfusion for another $2 \mathrm{~h}$. Control cells were cultured in 10\% FBS complete medium with 21\% oxygen. A gp130 and WSX-1 mRNA levels in HK-2 cells were determined by RT-PCR after I2h+R2h. B Determination of cell apoptosis by flow cytometry of annexin V/propidium iodide staining in HK-2 cells. C Representative images of Bcl-2, Bax and $\beta$-actin. D Densitometric analysis of Bcl-2 and Bax. Values are expressed as relative expression (arbitrary units). Data are expressed as means \pm SEM of more than 3 independent experiments in triplicate culture. ${ }^{*} p<0.05,{ }^{* *} p<0.01,{ }^{* * *} p<0.001$; ns, not significant.

(Fig. 1C). And the expression of kidney injury molecule 1 (Kim-1) significantly increased to 628.4-fold after I/R treatment, which decreased by 600 -fold by IL-27 treatment (Fig. 1D). PAS staining revealed that there was $57 \%$ less tubular injury in IL-27-treated kidneys as compared with that in vehicle-treated kidneys (Fig. 1E, F). Altogether, these data indicate that IL-27 treatment protects against I/R-induced kidney injury.

\section{IL-27 Treatment Attenuates Renal Inflammation after I/R}

Imbalanced production of proinflammatory and anti-inflammatory cytokines is one of the pathological causes of renal I/R-induced injury. To further investigate the renal protection mechanism of IL-27, mRNA expression of cytokines in kidneys was determined by RT-PCR. As illustrated in Figure 2A, I/R resulted in the overexpression of IL-17A, tumor necrosis factor- $\alpha$ (TNF- $\alpha$ ) and IL-6 (approx. 1,494.0-, 2.7- and 293.6-fold, respectively, compared to the sham group), and IL-27 administration significantly attenuated the production of these proinflammatory cytokines in the I/R group(approx. 1,148.6-, 1.2- and 150.2-fold, respectively, compared to the I/R with vehicle group). In contrast, IL-27 increased the expression of the anti-inflammatory cytokine IL-10 more than 150 -fold compared to the I/R with vehicle group. Collectively, these data strongly support the notion that IL-27 induces an anti-inflammatory response in I/R-induced AKI. 


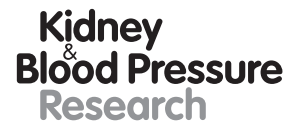

Research \begin{tabular}{l|l}
\hline Kidney Blood Press Res 2019;44:1453-1464 \\
\hline DOI: 10.1159/000503923 & $\begin{array}{l}\text { @ 2019 The Author(s). Published by S. Karger AG, Basel } \\
\text { www.karger.com/kbr }\end{array}$ \\
\hline
\end{tabular}

Zhou et al.: IL-27 in AKI

\section{IL-27 Reduces Apoptosis after I/R in Kidneys}

Apoptosis plays a vital role in I/R-induced tubular injury. To determine whether IL-27 treatment affects I/R-induced apoptosis, we measured the production of cleaved caspase- 3 in the kidneys. The production of cleaved caspase-3 was significantly upregulated by I/R and was reduced in kidneys that received IL-27 treatment (Fig. 2B). Moreover, proapoptotic protein Bax and antiapoptotic protein Bcl-2 in kidneys were analyzed by Western blotting. As illustrated in Figure 2D, the expression of Bax was significantly upregulated by I/R, which was reduced by IL-27 treatment. Meanwhile, the expression of Bcl-2 was enhanced by IL-27 treatment. The above data demonstrate that IL-27 treatment suppresses I/R-induced apoptosis in the kidneys.

\section{IL-27 Exerts Antiapoptotic Function in Renal Tubular Epithelial Cells}

As the death of renal tubular epithelial cells is a central mechanism of AKI, we investigated whether IL-27 could directly affect renal tubular epithelial cells. Our preliminary experiments showed the higher ratio of Bax/Bcl-2 in $\mathrm{I} 2 \mathrm{~h}+\mathrm{R} 2 \mathrm{~h}$ than that in $\mathrm{I} 2 \mathrm{~h}+\mathrm{R} 0 \mathrm{~h}$ (online suppl. Fig. 3). First, the expressions of IL-27 receptors, WSX-1 and gp130, in HK-2 cells were measured, and their expression significantly increased to 1.9- and 4.1-fold, respectively, after I2h+R2h treatment (Fig. 3A). And as shown in online supplementary Figure 2A, the expression of Hif- $\alpha$ significantly increased after $\mathrm{I} 2 \mathrm{~h}+\mathrm{R} 2 \mathrm{~h}$ treatment. Then HK-2 cells were treated with either recombinant human IL-27 or vehicle prior to I2h+R2h. Although IL-27 increased the expression of Hif- $\alpha$ (online suppl. Fig. 2B), flow cytometry analysis showed that IL-27 administration attenuated I2h+R2h-induced epithelial apoptosis by $72 \%$ (Fig. 3B). Meanwhile, IL-27 also reduced the Bax expression and increased Bcl-2 expression in the I2h+R2h group (Fig. 3C). The observation was further quantified and confirmed by densitometric analysis. The expression of Bax in IL-27-treated cells was $41 \%$ less than that in control cells after I2h+R2h treatment. IL-27 treatment increased Bcl-2 expression by $244 \%$ after $\mathrm{I} 2 \mathrm{~h}+\mathrm{R} 2 \mathrm{~h}$ treatment. And the ratio of Bax/Bcl-2 increased significantly $104 \%$ after I $2 \mathrm{~h}+\mathrm{R} 2 \mathrm{~h}$ treatment, which decreased $82 \%$ by IL-27 treatment (Fig. 3D). These data suggested that IL-27 exerts antiapoptotic effects in renal tubular epithelial cells under AKI conditions.

\section{Activation of STAT3 Is Essential for the IL-27-Mediated Protective Role}

IL-27 is reported to activate STAT3, which acts as a cell survival signal in AKI. We then investigated whether STAT3 activation is involved in the IL-27-mediated renoprotection. Increased expression of STAT3 and active pSTAT3 was found in renal tissues from I/R mice treated with IL-27 administration (Fig. 4A). Consistently, STAT3 and pSTAT3 in the HK-2 cells was also increased by IL-27 (Fig. 4B). As shown in Figure 4C, the STAT3 inhibitor Stattic blocked STAT3 activity in HK-2 cells. The antiapoptotic effects of IL-27 (as manifested by reduced Bax level and increased Bcl-2 level) were abrogated by Stattic. These data indicated that STAT3 activation is required for IL-27-mediated protection.

\section{Discussion}

The role of IL-27 in AKI is hardly known. In this study, we showed that recombinant IL-27 ameliorates renal I/R injury. The underlying mechanism relies to a large extend on IL-27 activating STAT3 in renal tubular epithelial cells (Fig. 5).

The heterodimeric cytokine IL-27 is mainly produced by active dendritic cells and macrophages, which are involved in the pathogenesis of kidney I/R [22]. In our study, we found that the expression of IL-27 in kidneys was increased after I/R, which may be due to the influx of 


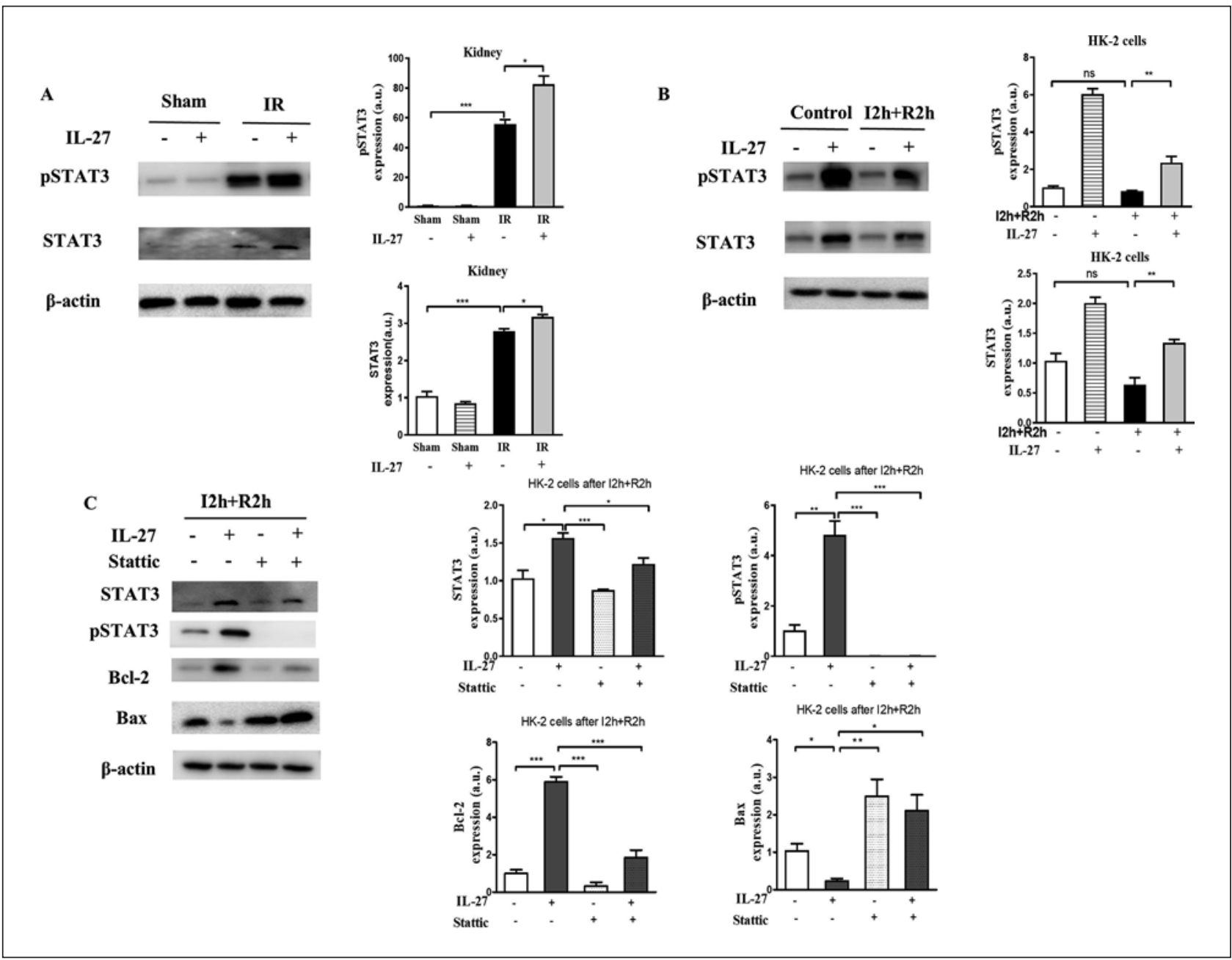

Fig. 4. Activation of STAT3 is essential for the IL-27-mediated protective effect. A STAT3 and pSTAT3 in renal tissue were analyzed $24 \mathrm{~h}$ after I/R. Similar results were obtained in 3 independent experiments with 6-7 mice per group. B The expression of STAT3 and pSTAT3 in renal tubular epithelial cells after $\mathrm{I} 2 \mathrm{~h}+\mathrm{R} 2 \mathrm{~h}$ was analyzed by Western blot. Densitometric analysis is shown on the right. Values are expressed as relative expression (arbitrary units). C The expression of STAT3, pSTAT3, Bcl-2 and Bax in I2h+R2h-treated HK-2 cells was assessed after IL-27 and STAT3 inhibitor Stattic treatment. Densitometric analysis of STAT3, pSTAT3, Bcl-2 and Bax is shown on the right. Values are expressed as relative expression (arbitrary units). Data are expressed as means \pm SEM of more than 3 independent experiments in triplicate culture. ${ }^{*} p<0.05,{ }^{* *} p<$ $0.01, * * * p<0.001 ;$ ns, not significant.

inflammatory cells in postischemia kidneys. Moreover, in our study we found that increased expression of IL-27 occurred at $24 \mathrm{~h}$, a late time point after I/R, which may only exert minimal benefits at this time point, and exogenous delivery of IL-27 at an early time point such as $1 \mathrm{~h}$ before ischemia may give more benefits.

In this study, we showed that recombinant IL-27 attenuates kidney inflammation. The two receptors of IL-27, gp130 and WSX-1, were expressed on T cells, and IL-27 has been shown to influence the function of T-cell subsets [7]. In a mouse model of multiple sclerosis, IL-27 inhibited the proliferation of T-helper type 17 cells and the production of IL-17 through the activation of STAT3 and STAT1 [23]. Yoshimura et al. [6] found that exogenous IL-27 suppressed IL-17 production in a dose-dependent manner. In AKI, T cells are involved in the 
Fig. 5. The mechanisms of IL-27mediated protective effects on renal I/R injury. Increased IL-27 in the kidney after injury leads to activation of STAT3, causing the upregulation of Bcl-2 and downregulation of Bax, thereby reducing apoptosis. Meanwhile, treatment with IL-27 can ameliorate renal inflammation after I/R.

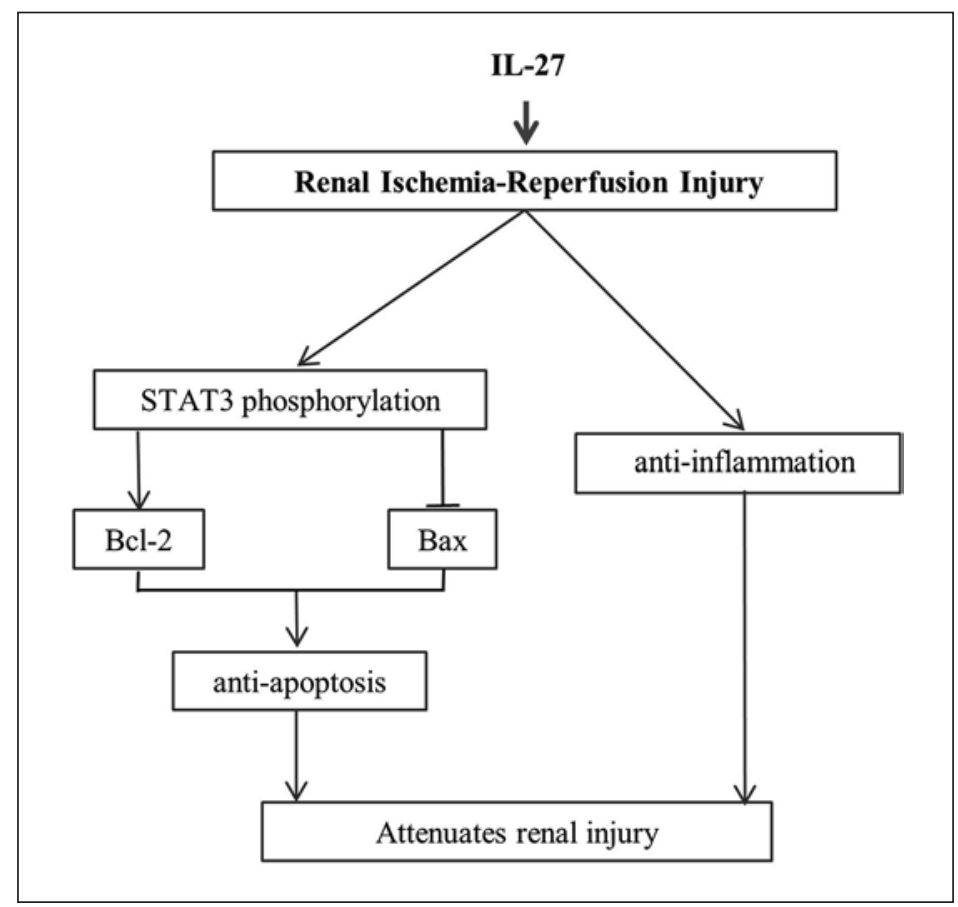

inflammatory process [24]. Although a direct effect of IL-27 on T cells in AKI is unknown, our present study provides evidence that exogenous IL-27 suppressed the expression of TNF- $\alpha$, IL-6 and IL-17A, which are T-cell-related cytokines. Thus, it is possible that IL-27 regulates $\mathrm{T}$-cell function and related cytokine secretion to attenuate AKI.

Besides inflammation, apoptosis is involved in pathological processes in kidneys after AKI [25]. Excessive apoptosis can lead to renal dysfunction and fibrosis [26]. Previous studies have shown that pharmacological inhibitors of apoptosis, such as 4-benzyl-2-methyl-1,2,4thiadiazolidine-3,5-dione and GSK3bs [27, 28], exert renoprotective effects. Our data show that IL-27 inhibited apoptosis in AKI by increasing the expression of the antiapoptotic protein $\mathrm{Bcl}-2$ and reducing the expression of the proapoptotic protein Bax in renal tubular epithelial cells.

The STAT signaling pathway plays an important role in renal disease $[29,30]$, which was activated in the mouse model of I/R-induced AKI. Previous studies showed that genetic deletion of STAT3 signaling exacerbated renal function loss and pathological injury [31]. IL-27 was reported to be an important upstream regulator of STAT3 [32]. In our study, recombinant IL-27 increased STAT3 expression and promoted STAT3 phosphorylation both in vivo and in vitro. Pharmacological inhibition of STAT3 inhibited Bcl-2 expression induced by IL-27 in vitro. Thus, our data demonstrated that IL-27 exerts its antiapoptotic effect through both upregulation and activation of STAT3.

To date, there is no therapeutic approach available to prevent or treat AKI. Our study demonstrated the protective role of IL-27 in AKI by reducing kidney inflammation and apoptosis. Further research with inhibition or knockout/knockdown of IL-27 will be required to confirm its protective effect. Moreover, some researchers have stressed the importance of STAT1 activation response to IL-27 [33], suggesting that the exact mechanism of IL-27 in AKI needs to be further investigated. 


\section{Acknowledgment}

This work is supported by grants from the National Natural Science Foundation of China (No. 81270850, 81470990, 8187030026) and the Science and Technology Commission of Shanghai Municipality (No. 16ZR1419400).

\section{Statement of Ethics}

All animal experiments were in accordance with Institutional Animal Care and Use Committee guidelines. The Animal Ethics Committee of Shanghai Jiao Tong Medical University approved all procedures.

\section{Disclosure Statement}

The authors have no conflicts of interest to declare.

\section{Author Contributions}

L.W. and F.D. conceived and designed the research; P.Z. and B.D. performed the research; P.Z. and B.D. analyzed the data; P.Z., B.D. and M.W. wrote the paper.

\section{References}

1 Lameire N, Van Biesen W, Vanholder R. The changing epidemiology of acute renal failure [J]. Nat Clin Pract Nephrol. 2006 Jul;2(7):364-77.

2 Lameire NH, Bagga A, Cruz D, De Maeseneer J, Endre Z, Kellum JA, et al. Acute kidney injury: an increasing global concern. Lancet. 2013 Jul;382(9887):170-9.

3 Leaf DE, Rajapurkar M, Lele SS, Mukhopadhyay B, Rawn JD, Frendl G, et al. Increased plasma catalytic iron in patients may mediate acute kidney injury and death following cardiac surgery. Kidney Int. 2015 May;87(5): 1046-54.

4 Pflanz S, Timans JC, Cheung J, Rosales R, Kanzler H, Gilbert J, et al. IL-27, a heterodimeric cytokine composed of EBI3 and p28 protein, induces proliferation of naive CD4+ T cells. Immunity. 2002 Jun;16(6):779-90.

5 Findlay EG, Greig R, Stumhofer JS, Hafalla JC, de Souza JB, Saris CJ, et al. Essential role for IL-27 receptor signaling in prevention of Th1-mediated immunopathology during malaria infection. J Immunol. 2010 Aug; 185(4):2482-92.

6 Yoshimura T, Takeda A, Hamano S, Miyazaki Y, Kinjyo I, Ishibashi T, et al. Two-sided roles of IL-27: induction of Th1 differentiation on naive CD4+ T cells versus suppression of proinflammatory cytokine production including IL-23-induced IL-17 on activated CD4+ T cells partially through STAT3-dependent mechanism. J Immunol. 2006 Oct;177(8):5377-85.

7 Mchedlidze T, Kindermann M, Neves AT, Voehringer D, Neurath MF, Wirtz S. IL-27 suppresses type 2 immune responses in vivo via direct effects on group 2 innate lymphoid cells. Mucosal Immunol. 2016 Nov;9(6):138494.

8 Phan WL, Huang YT, Ma MC. Interleukin-27 protects cardiomyocyte-like H9c2 cells against metabolic syndrome: role of STAT3 signaling. BioMed Res Int. 2015;2015:689614.

9 Ma MC, Wang BW, Yeh TP, Wu JL, Chung TH, Tsui K, et al. Interleukin-27, a novel cytokine induced by ischemiareperfusion injury in rat hearts, mediates cardioprotective effects via the gp130/STAT3 pathway. Basic Res Cardiol. 2015 May;110(3):22-34.

10 Zhu H, Lou C, Liu P. Interleukin-27 ameliorates coxsackievirus-B3-induced viral myocarditis by inhibiting Th17 cells. Virol J. 2015 Nov;12(1):189-96.

11 Pickens SR, Chamberlain ND, Volin MV, Mandelin AM 2nd, Agrawal H, Matsui M, et al. Local expression of interleukin-27 ameliorates collagen-induced arthritis. Arthritis Rheum. 2011 Aug;63(8):2289-98.

12 Su Y, Yao H, Wang H, Xu F, Li D, Li D, et al. IL-27 enhances innate immunity of human pulmonary fibroblasts and epithelial cells through upregulation of TLR4 expression. Am J Physiol Lung Cell Mol Physiol. 2016 Jan; 310(2):L133-41. 
13 Tanida S, Yoshitomi H, Ishikawa M, Kasahara T, Murata K, Shibuya H, et al. IL-27-producing CD14(+) cells infiltrate inflamed joints of rheumatoid arthritis and regulate inflammation and chemotactic migration. Cytokine. 2011 Aug;55(2):237-44.

14 Shukla P, Mansoori MN, Kakaji M, Shukla M, Gupta SK, Singh D. Interleukin 27 (IL-27) Alleviates Bone Loss in Estrogen-deficient Conditions by Induction of Early Growth Response-2 Gene. J Biol Chem. 2017 Mar;292(11): 4686-99.

15 Yang B, Suwanpradid J, Sanchez-Lagunes R, Choi HW, Hoang P, Wang D, et al. IL-27 facilitates skin wound healing through induction of epidermal proliferation and host defense. J Invest Dermatol. 2017 May;137(5): 1166-75.

16 Watzlawick R, Kenngott EE, Liu FD, Schwab JM, Hamann A. Anti-inflammatory effects of IL-27 in zymosaninduced peritonitis: inhibition of neutrophil recruitment partially explained by impaired mobilization from bone marrow and reduced chemokine levels. PLoS One. 2015;10(9):e0137651.

17 Oh DJ, Dursun B, He Z, Lu L, Hoke TS, Ljubanovic D, et al. Fractalkine receptor (CX3CR1) inhibition is protective against ischemic acute renal failure in mice. Am J Physiol Renal Physiol. 2008 Jan;294(1):F264-71.

18 Jiang M, Liu K, Luo J, Dong Z. Autophagy is a renoprotective mechanism during in vitro hypoxia and in vivo ischemia-reperfusion injury. Am J Pathol. 2010 Mar;176(3):1181-92.

19 Dorosz SA, Ginolhac A, Kähne T, Naumann M, Sauter T, Salsmann A, et al. Role of calprotectin as a modulator of the IL27-mediated proinflammatory effect on endothelial cells. Mediators Inflamm. 2015;2015(3):737310.

20 Kachroo P, Lee MH, Zhang L, Baratelli F, Lee G, Srivastava MK, et al. IL-27 inhibits epithelial-mesenchymal transition and angiogenic factor production in a STAT1-dominant pathway in human non-small cell lung cancer. J Exp Clin Cancer Res. 2013 Nov;32(1):97-120.

21 Peters A, Fowler KD, Chalmin F, Merkler D, Kuchroo VK, Pot C. IL-27 induces Th17 differentiation in the absence of STAT1 signaling. J Immunol. 2015 Nov;195(9):4144-53.

22 Jones SA, Jenkins BJ. Recent insights into targeting the IL-6 cytokine family in inflammatory diseases and cancer. Nat Rev Immunol. 2018 Dec;18(12):773-89.

23 Li L, Huang L, Vergis AL, Ye H, Bajwa A, Narayan V, et al. IL-17 produced by neutrophils regulates IFN-gammamediated neutrophil migration in mouse kidney ischemia-reperfusion injury. J Clin Invest. 2010 Jan; 120(1): 331-42.

24 Li L, Huang L, Sung SS, Lobo PI, Brown MG, Gregg RK, et al. NKT cell activation mediates neutrophil IFN-gamma production and renal ischemia-reperfusion injury. J Immunol. 2007 May;178(9):5899-911.

25 Kelly KJ, Plotkin Z, Dagher PC. Guanosine supplementation reduces apoptosis and protects renal function in the setting of ischemic injury. J Clin Invest. 2001 Nov;108(9):1291-8.

26 Havasi A, Borkan SC. Apoptosis and acute kidney injury. Kidney Int. 2011 Jul;80(1):29-40.

27 Zhou Y, Mao H, Li S, Cao S, Li Z, Zhuang S, et al. HSP72 inhibits Smad3 activation and nuclear translocation in renal epithelial-to-mesenchymal transition. J Am Soc Nephrol. 2010 Apr;21(4):598-609.

28 Wang Z, Havasi A, Gall J, Bonegio R, Li Z, Mao H, et al. GSK3beta promotes apoptosis after renal ischemic injury. J Am Soc Nephrol. 2010 Feb;21(2):284-94.

29 Wu M, Chen M, Jing Y, Gu J, Mei S, Yao Q, et al. The C-terminal tail of polycystin-1 regulates complement factor B expression by signal transducer and activator of transcription 1. Am J Physiol Renal Physiol. 2016 Jun; 310(11):F1284-94.

30 Jing Y, Wu M, Zhang D, Chen D, Yang M, Mei S, et al. Triptolide delays disease progression in an adult rat model of polycystic kidney disease through the JAK2-STAT3 pathway. Am J Physiol Renal Physiol. 2018 Sep; 315(3):F479-86.

31 Dube S, Matam T, Yen J, Mang HE, Dagher PC, Hato T, et al. Endothelial STAT3 Modulates Protective Mechanisms in a Mouse Ischemia-Reperfusion Model of Acute Kidney Injury. J Immunol Res. 2017;2017:4609502.

32 Fabbi M, Carbotti G, Ferrini S. Dual roles of IL-27 in cancer biology and immunotherapy. Mediators Inflamm. 2017;2017(42):3958069.

33 Lucas S, Ghilardi N, Li J, de Sauvage FJ. IL-27 regulates IL-12 responsiveness of naive CD4+ T cells through Stat1-dependent and -independent mechanisms. Proc Natl Acad Sci USA. 2003 Dec;100(25):15047-52. 